\title{
$\angle S$ Research Square \\ The prognostic significance of APOA1 mRNA and protein in kidney renal clear cell carcinoma
}

\section{Xiulan Peng ( $\nabla$ Dr.Xiulan@whu.edu.cn )}

The second affiliated hospital of jianghan university https://orcid.org/0000-0003-1835-0992

\section{Guoguang Xiong}

The second affiliated hospital of Jianghan University

\section{Jingtao Li}

The second affiliated hospital of Jianghan university

\section{Yugang $\mathrm{Hu}$}

Renmin Hospital of Wuhan University: Wuhan University Renmin Hospital

\section{Anbing He}

The second affiliated hospital of Jianghan university

\section{Xia Wang}

the second affiliated hospital of jianghan university

\section{Renfeng Luo}

Jianghan University

\section{Research Article}

Keywords: Apolipoprotein A1(APOA1), kidney renal clear cell carcinoma, bioinformatic analysis, overall survival, prognostic significance

Posted Date: February 23rd, 2022

DOI: https://doi.org/10.21203/rs.3.rs-1380075/v1

License: (c) (i) This work is licensed under a Creative Commons Attribution 4.0 International License. Read Full License 


\section{Abstract \\ Background}

We attempted to evaluate the clinical and prognostic significance of APOA1 mRNA and protein in kidney renal clear cell carcinoma (KIRC).

\section{Methods}

Clinical data along with RNA-sequencing data were downloaded from UCSC Xena. The Human Protein Atlas (HPA) database was searched to reveal the expression profiles of APOA1 protein in KIRC tissues and the normal renal tissues. TIMER database was applied to determine the correlations of APOA1 with immune cells and PD-1.PD-L1 in KIRC. 91 cases of KIRC patients and 93 cases of healthy controls from Renmin Hospital of Wuhan University were enrolled for clinical validation.

\section{Results}

Levels of APOA1 mRNA in KIRC tissues ( $N=533$ ) seemed to be lower than the levels in normal renal tissues $(N=72)$, but the difference was statistically insignificant $(P=0.545)$. Multivariate Cox results reveled that low expression of APOA1 mRNA was a reliable marker for favorable OS (HR $=1.66, P=0.037)$ and DFS $(H R=1.65, P=0.047)$, and APOA1 hypermethylation was a reliable biomarker for worse OS (HR $=2.1, P=0.001)$ rather than DFS $(H R=1.12, P=0.624)$ in KIRC patients. The HPA database indicated that APOA1 protein expression was down-regulated in renal cancerous tissues compared with that in the normal renal tissues. Moreover, concentrations of serum APOA1 protein were markedly decreased in KIRC patients compared to healthy controls $(P<0.01)$, and low levels of APOA1 protein predicted less favorable OS than those with high levels $(H R=2.84, P=0.0407)$. Finally, APOA1 was negatively correlated with various immune cells infiltrates and PD-L1 expression $(r=-0.283, P=2.74 \mathrm{e}-11)$ based on TIMER database.

\section{Conclusion}

Low levels of APOA1 mRNA or high levels of serum APOA1 protein predict favorable survival in KIRC patients. Our study has provided novel insights for identifying a novel prognostic index with great clinical utility and therapeutic target for KIRC.

\section{Background}

The American Cancer Society predicted that 73,750 individuals in the United States would be newly diagnosed with renal carcinoma by the end of 2020 (1). The most common subtype of renal cancer is kidney renal clear cell carcinoma (KIRC), which is also the most common malignancy of the urinary system (2). For patients with KIRC and only local invasion, surgical resection remains the primary and 
most effective treatment. For patients with KIRC who have remote metastases, however, treatment is much more complex as KIRC is insensitive to traditional chemotherapy or radiotherapy (3). Therefore, understanding the etiology of KIRC is necessary so that novel prognostic or therapeutic biomarkers can be discovered.

Although KIRC has been extensively studied, knowledge of its molecular mechanisms and accurate predictions concerning prognosis are limited. KIRC exhibits significant molecular heterogeneity (4) involving multiple changes in gene expression. With the aid of bioinformatics, a series of key genes associated with renal cancer have been identified as potential biomarkers for diagnosis, survival prediction, and as therapeutic targets (5-7). However, as most of these biomarkers are not measured routinely in clinical practice, they are not clinically useful. Therefore, identifying a novel prognostic marker that is easily accessible clinically and can accurately predict survival is essential for the management of patients with KIRC.

Apolipoprotein A1 (APOA1), a common index of blood lipids, is thought to play an essential role in the occurrence and progression of cardiovascular diseases (8-10). Recently, it has been reported that APOA1 is associated with colorectal $(11,12)$, ovarian $(13)$, breast $(14)$, and liver cancer (15) survival. Moreover, two studies have demonstrated that preoperative APOA1 concentration is an independent factor affecting survival in patients with metastatic renal cell cancer $(16,17)$. However, no study has comprehensively assessed the association between APOA1 and primary KIRC. In this study, therefore, we applied bioinformatics to explore APOA1 mRNA and protein levels in terms of expression and prognosis in patient with KIRC. We also retrospectively collected the clinical data of 91 patients with KIRC from the Renmin Hospital of Wuhan University (RHWU) to investigate the expression of the APOA1 protein and the associated survival profiles.

\section{Methods}

\section{UCSC Xena}

UCSC Xena (http://xena.ucsc.edu/\#overview) is an online exploration website for the visualization and analysis of cancer genomic data based on The Cancer Genomic Atlas (TCGA) database (18). For this study, we extracted APOA1 expression data from 533 patients with KIRC and 72 subjects with normal renal tissue using the visualization module. The data sets module was used to download clinical information from the TCGA-KIRC dataset. Subsequently, the visualization module was used again to determine any association between APOA1 mRNA and APOA1 DNA methylation.

\section{UALCAN}

UALCAN is a very friendly web tool (http://ualcan.path.uab.edu/index.html) that can be used to accurately analyze omics data (TCGA and MET500) (19). In this study, we used the UALCAN tool to analyze the differential expression of APOA1 mRNA in KIRC tissue compared to normal renal specimens. 
To do this, we entered the APOA1 gene into the TCGA gene analysis module, selected KIRC, and then obtained the differential expressions of APOA1 both in KIRC tissues and in normal specimens.

\section{LinkedOmics}

The LinkedOmics website is an easily accessible research platform that provides a series of data analyses based on the TCGA dataset (20). In our study, we searched this database to identify the genes that were most significantly associated with APOA1 expression in KIRC tissue through the LinkFinder module, and then selected these genes for enrichment analysis via the LinkerInterpreter module and the Gene Set Enrichment Analysis (GSEA) tool.

\section{The human protein atlas}

The Human Protein Atlas (HPA) database is an open-source database of all types of human proteins found in normal and cancerous tissues (21). In the current study, the protein expression module was used to investigate the protein levels of APOA1 in normal renal tissues and KIRC tissues, as detected by immunohistochemistry (IHC)in the HPA database. Two anti-APOA1 antibodies (HPA046715 and CAB016778) were used to determine the levels of APOA1 protein expression in normal renal and KIRC tissues. HPA046715 is a polyclonal antibody derived from rabbits and produced by Sigma-Aldrich, and CAB016778 is a monoclonal antibody derived from mice and produced by BioPorto Diagnostics A/S.

\section{TIMER database}

The TIMER database (https://cistrome.shinyapps.io/timer/) is a valuable resource for the comprehensive analysis of immune infiltrates in various types of cancer (22). This database provided us with six immune infiltrates estimated by the TIMER algorithm. The correlation between APOA1 expression and infiltrating immune cells was detected using the gene module and the correlation module was used to determine the association between APOA1 and other genes (PD-1/PD-L1). The correlation module was then used to determine the association between APOA1 and immune cell markers.

\section{Clinical data from the RHWU cohort}

We retrospectively enrolled 91 patients diagnosed with KIRC who were treated at RHWU between January 2016 and December 2019. An additional 93 healthy individuals were enrolled as controls. The study outline was reviewed and smoothly approved by the ethics committee of the RHWU. We obtained serum APOA1 protein levels and other clinical data, such as sex, age at diagnosis, tumor grade, tumor site, $T$ stage, $\mathrm{N}$ stage, $\mathrm{M}$ stage, and TNM stage from the electronic medical records. Follow-up was performed by telephone interview until August 31,2020. Overall survival (OS) was calculated as the interval between surgical resection of the renal tumor and the last follow-up or death from any cause. 


\section{Statistical analysis}

Continuous and categorical variables are expressed as means with standard deviations and numbers with percentages, respectively. For categorical variables, differences between the two groups were analyzed using the chi-square or Fisher's exact test. For continuous variables, differences were determined using the Student's $t$-test or nonparametric test. Receiver operating characteristic (ROC) curves were drawn to assess AP01 mRNA and serum protein levels as potential diagnostic predictors. Kaplan-Meier curves were plotted to assess APOA1 mRNA and protein levels as prognostic indicators, and the log-rank test was used to assess the difference in survival between the two groups. Cox regression analyses were performed to determine whether APOA1 mRNA or APOA1 protein levels were independent biomarkers of prognosis in patients with KIRC. The correlation between the two genes was determined using the Spearman correlation test. All statistical analyses were performed using SPSS 20.0. All tests were performed on both sides, and a $P$ value $<0.05$ was regarded as statistically significant.

\section{Results}

\section{APOA1 mRNA expression and DNA methylation in KIRC}

The UALCAN tool was used to analyze the expression profiles of APOA1 mRNA in KIRC tissues compared to that in normal tissues. The results (Fig. 1A) indicated that the levels of APOA1 mRNA in KIRC tissues $(N=533)$ were lower than that in normal renal tissues $(N=72)$, but the difference was not significant after statistical analysis $(P=0.545)$. The ROC curve was used to assess the potential role of APOA1 as a diagnostic marker in patients with KIRC. As shown in Figure S1, APOA1 was acceptable at distinguishing KIRC tissue from normal tissue, with an area under the curve (AUC) of 0.708 (95\% Cl: 0.647-0.769). We then used the UCSC Xena tool to study the association between APOA1 expression and DNA methylation in KIRC tissue. As shown in Fig. 1B, a weak association was observed between APOA1 expression and DNA methylation. A correlation analysis (Table S1) was performed to find any associations between APOA1 expression and certain APOA1 DNA CpG sites. As illustrated in Figure S2, three APOA1 CpG sites (cg25987102, cg00142925, and cg19299755) were significantly associated with APOA1 expression. The distribution plot (Fig. 1C) of APOA1 DNA CpG sites also showed that the levels of APOA1 methylation between KIRC tissues and normal tissues were significantly different. As shown in Table S2, a chi-square analysis revealed that the expression of APOA1 mRNA was significantly associated with histological grade $(P=0.042)$ and white blood cell count $(P=0.047)$. Similarly, levels of APOA1 DNA methylation were markedly correlated with $\mathrm{N}$ stage $(P=0.031)$, M stage $(P=0.001)$, and TNM stage $(P=0.045)$, indicating that APOA1 DNA methylation may be a novel indicator of metastasis in patients with KIRC.

\section{Prognostic role of APOA1 mRNA in KIRC}

Survival analyses were performed to reveal the prognostic significance of APOA1 mRNA and APOA1 DNA methylation for KIRC. Kaplan-Meier curves (Fig. 2) showed that low expression of APOA1 mRNA was 
associated with favorable OS (HR $=1.59, P=0.0022)$ and disease-free survival (DFS) $(H R=1.61, P=$ 0.015). Similarly, survival analyses also demonstrated that APOA1 DNA hypermethylation was correlated with unfavorable OS (HR=1.55, $P=0.0331)$ and DFS $(H R=1.61, P=0.015)$. Cox regression analyses were then performed to identify independent prognostic indicators for KIRC. The results showed that high expression of APOA1 mRNA was a reliable marker of unfavorable OS (HR $=1.66, P=0.037)$ and DFS (HR $=1.65, P=0.047)$, and APOA1 DNA hypermethylation was a reliable biomarker of unfavorable OS (HR = 2.1, $P=0.001$ ) but was not a significant biomarker for DFS (HR=1.12, $P=0.624)$ (Table 1). In summary, APOA1 mRNA was determined to be a reliable indicator for predicting survival (OS and DFS) among patients with KIRC, while APOA1 DNA methylation may be a novel biomarker for predicting OS. 
Table 1

Multivariable analysis of APOA1 mRNA expression/methylation for overall survival /diseasefree survival

\begin{tabular}{|c|c|c|c|c|c|c|}
\hline \multirow[t]{2}{*}{ Variables } & \multicolumn{3}{|c|}{ Overall survival } & \multicolumn{3}{|c|}{ Disease-free survival } \\
\hline & HR & $95 \% \mathrm{Cl}$ & $P$ value & HR & $95 \% \mathrm{Cl}$ & $P$ value \\
\hline \multicolumn{7}{|c|}{ Age (years) } \\
\hline$\geq 50$ & 1.74 & $1.09-2.78$ & 0.021 & & & \\
\hline$<50$ & Ref. & - & - & & & \\
\hline \multicolumn{7}{|l|}{ Gender } \\
\hline Male & & & & 1.22 & $0.77-1.94$ & 0.403 \\
\hline Female & & & & Ref. & - & - \\
\hline \multicolumn{7}{|l|}{ Laterality } \\
\hline Left & 1.56 & $0.99-2.44$ & 0.053 & 1.82 & $1.19-2.80$ & 0.006 \\
\hline Right & Ref. & - & - & Ref. & - & - \\
\hline \multicolumn{7}{|c|}{ Longest dimension } \\
\hline$\geq 1.5$ & 1.07 & $0.69-1.69$ & 0.755 & & & \\
\hline$<1.5$ & Ref. & - & - & & & \\
\hline \multicolumn{7}{|c|}{ Histologic Grade } \\
\hline $\mathrm{G} 1+\mathrm{G} 2$ & Ref. & - & - & Ref. & - & - \\
\hline $\mathrm{G} 3+\mathrm{G} 4$ & 1.72 & $1.00-2.98$ & 0.052 & 2.45 & $1.44-4.17$ & 0.001 \\
\hline \multicolumn{7}{|l|}{ T stage } \\
\hline $\mathrm{T} 1+\mathrm{T} 2$ & Ref. & - & - & Ref. & - & - \\
\hline $\mathrm{T} 3+\mathrm{T} 4$ & 1.86 & $0.80-4.34$ & 0.152 & 1.18 & $0.61-2.29$ & 0.627 \\
\hline \multicolumn{7}{|l|}{$N$ stage } \\
\hline $\mathrm{NO}+\mathrm{NX}$ & Ref. & - & - & Ref. & - & - \\
\hline $\mathrm{N} 1$ & 6.50 & $2.24-18.9$ & 0.001 & 2.83 & $0.93-8.59$ & 0.066 \\
\hline \multicolumn{7}{|l|}{ M stage } \\
\hline$M 0+M X$ & Ref. & - & - & Ref. & - & - \\
\hline M1 & 3.07 & $1.71-5.450$ & $<0.001$ & 3.43 & $2.04-5.76$ & $<0.001$ \\
\hline
\end{tabular}




\begin{tabular}{|c|c|c|c|c|c|c|}
\hline \multirow[t]{2}{*}{ Variables } & \multicolumn{3}{|c|}{ Overall survival } & \multicolumn{3}{|c|}{ Disease-free survival } \\
\hline & HR & $95 \% \mathrm{Cl}$ & $P$ value & HR & $95 \% \mathrm{Cl}$ & $P$ value \\
\hline$I+I I$ & Ref. & - & - & Ref. & - & - \\
\hline III + IV & 1.69 & $0.92-3.10$ & & 5.06 & $2.15-11.87$ & $<0.001$ \\
\hline \multicolumn{7}{|l|}{ Radiation } \\
\hline Yes & & & & 3.65 & $0.82-16.36$ & 0.091 \\
\hline No & & & & Ref. & - & - \\
\hline \multicolumn{7}{|c|}{ Neoadjuvant treatment } \\
\hline Yes & 1.10 & $0.32-3.82$ & 0.877 & & & \\
\hline No & Ref. & - & - & & & \\
\hline \multicolumn{7}{|c|}{ APOA1expression } \\
\hline Low & Ref. & - & - & Ref. & - & - \\
\hline High & 1.66 & $1.05-2.62$ & 0.031 & 2.10 & $1.34-3.28$ & 0.001 \\
\hline \multicolumn{7}{|c|}{ APOA1 methylation } \\
\hline Low & Ref. & - & - & Ref. & - & - \\
\hline High & 1.65 & $1.01-2.70$ & 0.047 & 1.12 & $0.72-1.74$ & 0.624 \\
\hline
\end{tabular}

\section{Expression of the APOA1 protein based on the HPA database}

To analyze APOA1 protein expression patterns in cancerous compared to normal renal tissues, the HPA database was systematically searched. Two kinds of APOA1 antibodies (CAB016778 and HPA046715) were used for immunohistochemistry. Three cases of normal renal tissue were strongly stained with the two antibodies. However, 12 of the cases of renal cancerous tissues were not positive for CAB016778, and only one case of renal cancerous tissue showed very weak positive staining for HPA046715. Representative images stained with the APOA1 antibodies are shown in Fig. 3. We concluded that APOA1 protein expression was downregulated in renal cancerous tissues compared to normal renal tissues.

\section{Levels of serum APOA1 protein in patients with KIRC}

We collected clinical data from the RHWU cohort to explore the clinical value and prognostic significance of serum APOA1 protein levels in patients with KIRC. The findings (Fig. 4A) suggested that concentrations 
of serum APOA1 protein levels were markedly decreased in patients with KIRC compared to healthy controls $(P<0.01)$. ROC analysis showed that serum APOA1 protein levels exhibited good diagnostic ability (AUC $=0.707,95 \% \mathrm{Cl}: 0.644-0.790)$ to differentiate patients with KIRC from healthy controls (Fig. 4B). Moreover, serum APOA1 protein levels were significantly associated with a panel of clinical features (Fig. 5), including histological grade $(P=0.0047)$, T stage $(P<0.0001)$, M stage $(P=0.0001)$, and TNM stage ( $P=0.0138)$. The Kaplan-Meier plot (Fig. $4 C)$ revealed a close correlation between low levels of serum APOA1 protein and unfavorable OS $(\mathrm{HR}=2.84, P=0.0407)$ in the 91 patients with KIRC. Multivariate Cox analysis indicated that serum APOA1 protein level was a reliable index for predicting OS $(\mathrm{HR}=2.91, P=0.023)$ in patients with KIRC (Fig. 4D).

\section{Enrichment analysis}

The LinkedOmics database was used to identify the genes that were most significantly correlated with APOA1 expression in KIRC. The volcano map (Fig. 6A) shows all the genes that were correlated with APOA1 expression. The positively correlated genes are displayed in Fig. 6B, and the negatively associated genes are listed in Fig. 6C. Subsequently, we utilized the GSEA module from the LinkedOmics database for enrichment analysis. The GO analysis (Table S3) demonstrated that the genes that were co-expressed with APOA1 were mostly implicated in mitochondrial gene expression, humoral immune response, protein targeting, ncRNA processing, and the nucleoside monophosphate metabolic process, among others. A KEGG analysis was then performed, which demonstrated that these genes were mainly involved in the phosphatidylinositol signaling system, thyroid hormone signaling pathway, FoxO signaling pathway, purine metabolism, spliceosome, drug metabolism, and systemic lupus erythematosus. A detailed description of the KEGG analysis is shown in Table S4.

\section{Correlation between APOA1 and immune cells}

After APOA1's involvement in the human immune response was revealed by the enrichment analysis, we searched the TIMER database to reveal any correlation between APOA1 expression and infiltrating immune cells and tumor purity. As shown in Figure S3, APOA1 expression was negatively correlated with tumor purity $\left(r=-0.152, P=1.04 \mathrm{e}^{-3}\right)$, CD8 $+\mathrm{T}$ cells $\left(r=-0.126, P=8.51 \mathrm{e}^{-3}\right)$, macrophages $(r=-0.144, P=$

$\left.2.19 \mathrm{e}^{-3}\right)$, neutrophils $\left(\mathrm{r}=-0.164, P=4.31 \mathrm{e}^{-3}\right)$, and dendritic cells $\left(\mathrm{r}=-0.101, P=3.17 \mathrm{e}^{-3}\right)$. No associations were observed between APOA1 expression and B cells or CD $4+T$ cells. We then used the correlation module to evaluate the correlation between APOA1 expression and gene markers of immune cell subtypes. As shown in Table S5, we noticed that APOA1 expression was correlated with B cells (CD19, CD79A) and Th2 cells (GATA3, STATA5A, STATA6). Due to the increasingly important role of immunotherapy in the treatment of renal cell carcinoma $(23,24)$, we also determined the relationship between APOA1 DNA expression in KIRC with PD-1 and PD-L1. We observed a negative correlation between APOA1 expression and PD-L1 expression $\left(r=-0.283, P=2.74 \mathrm{e}^{-11}\right)$, while no association was observed between APOA1 and PD-1 $\left(r=0.085, P=5.1 e^{-2}\right)$ (Figure S4). 


\section{Discussion}

Renal cancer is a common malignancy in both male and female patients, with a high incidence and mortality rate (25). However, the clinical use of molecular biomarkers associated with prognosis in KIRC is limited. Therefore, our study was designed to explore the clinical applicability of APOA1, which is easily measurable in clinical practice, for predicting survival in patients with KIRC. This study revealed that the expression of APOA1 mRNA is closely related to a series of clinical features. Moreover, survival analyses showed that low expression of APOA1 mRNA was an independent indicator of favorable OS and DFS in patients with KIRC. The expression of APOA1 protein was lower in KIRC tissue than in normal renal tissue. We found a similar trend in the clinical data, with low levels of serum APOA1 protein associated with longer OS in patients with KIRC. These results indicate that APOA1 may be a promising biomarker for predicting survival among patients with KIRC.

APOA1 is a major protein constituent of high-density lipoprotein (26) and has been reported to be involved in angiogenesis, tumor growth, and metastasis $(27,28)$. There are several kinds of singlenucleotide polymorphisms in the APOA1 gene, and an increased risk of renal cancer has been associated with the APOA1 -75 A allele and the APOA1 -75 AA genotype (29). Moreover, APOA1 has been correlated with survival in patients with cancer. Luo et al. (30) reported APOA1 as a novel prognostic index for risk definition in non-metastatic nasopharyngeal carcinoma. A recent clinical study, which included 144 patients with colorectal cancer, revealed that low levels of serum APOA1 protein were associated with systemic inflammation, advanced stage, and unfavorable survival (12). Wang et al. (31) suggested that APOA1 protein levels should be assessed pre-treatment as a predictor of OS in patients with esophageal squamous cell carcinoma. To our knowledge, this is the first study to uncover the association between low levels of APOA1 mRNA and hypomethylation of APOA1 with favorable survival in patients with KIRC. In this study, we also analyzed the relationship between serum APOA1 levels and prognosis in patients with KIRC. Since levels of the APOA1 protein can easily be measured in blood plasma, increased levels could be used as a biomarker of favorable survival in patients with KIRC.

We noticed that APOA1 mRNA expression was lower in the $533 \mathrm{KIRC}$ tissue samples than in the 72 normal tissue samples; however, the difference was not statistically significant. In terms of the distribution of APOA1 DNA methylation, we observed that the methylation levels of four important CpG sites (cg26734040, cg19324627, cg03010018, and cg24984312) were much higher in KIRC tissue than in normal specimens. Thus, we speculated that DNA methylation of APOA1 might partially account for the low expression of APOA1 mRNA in KIRC tissues. Subsequently, we searched the HPA database to determine whether APOA1 protein expression between KIRC and normal renal tissues was different. Upon performing immunohistochemical staining, we found that APOA1 protein was strongly stained in normal renal tissues, while it was weakly stained in KIRC tissues. Finally, we collected clinical data from our hospital to determine the differential expression of serum APOA1 protein. We noticed that the levels of serum APOA1 protein were lower in patients with KIRC compared with healthy controls. Hence, we conservatively concluded that APOA1 might act as a potential tumor suppressor gene in KIRC. 
Renal cancer is an immunogenic carcinoma associated with immune cell dysfunction and subsequent inability to control tumor growth (32). Furthermore, one study found that APOA1 could inhibit monocyte recruitment and macrophage chemotaxis and thus be involved in the immune response (33). Mao et al. (34) discovered that overexpression of APOA1 can significantly reduce the COX-2-induced inflammatory response in hepatocytes. When we analyzed the genes that were co-expressed with APOA1 in the GSEA analysis, we found that APOA1 also participates in the human immune response in KIRC tissues. Therefore, we searched the TIMER database to analyze the association between APOA1 and immune infiltrates in KIRC. APOA1 expression was also negatively correlated with an abundance of macrophages, CD8 + T cells, neutrophils, and dendritic cells. Due to the close association between APOA1 and immune cells in KIRC, we further studied the association between APOA1 and PD1/PD-L1 to determine whether APOA1 could be a target of immunotherapy for KIRC. We observed a negative correlation between APOA1 expression and PD-L1 expression, while no association was observed between APOA1 and PD-1. Previously, PD-L1 has been reported to act as an accurate biomarker for KIRC (35), and the relationship between PD-L1 and survival was more robust than that of PD-1 (36). Moreover, agents targeting PD-L1 have had promising effects in renal cell carcinoma (32). Immunosuppression and angiogenesis are closely linked in the renal cancerous microenvironment and are important mechanisms by which KIRC proliferates and metastasizes (37). The inverse association between APOA1 expression and PD-L1 implies that APOA1 plays a tumor-suppressive role in KIRC, and may be a novel therapeutic target for KIRC.

APOA1 belongs to the family of apolipoproteins A1/A4/E and plays an essential role in lipid metabolism (38). Cristina et al. (11) found that the overexpression of APOA1 can reduce the malignant features of colorectal cancer by regulating the export of cholesterol and downregulating the expression of COX-2. Therefore, apabetalone, which is a synthetic stimulator of APOA1, is a recognized inhibitor against colorectal cancer. The authors (11) concluded that APOA1, through its modulation of lipid metabolism, could be a new therapeutic target for colorectal cancer treatment. APOA1 has also been implicated in the progression of renal cell cancer through the regulation of lipid metabolism (39), and inhibition of APOA1 might be effective for the treatment of renal cell cancer. Further studies of APOA1 stimulators, such as apabetalone, for treating renal cell cancer are urgently needed.

However, our analysis has a few shortcomings. First, we could not validate the clinical and prognostic significance of APOA1 mRNA in another public dataset for technical reasons. Although we further verified the prognostic value of serum APOA1 protein levels using data from our hospital, the sample size of patients with KIRC used in our study was relatively small. Moreover, we focused on bioinformatic analysis and clinical data, and no biomedical experiments were implemented to understand the in-depth mechanism of APOA1 in the progression in KIRC and the development of metastases. Therefore, a series of functional experiments to elucidate the role of APOA1 in KIRC would be valuable in the future.

\section{Conclusions}


In summary, we comprehensively investigated the expression and prognostic profiles of APOA1 mRNA and protein levels in KIRC. APOA1 protein levels showed excellent diagnostic capability. Our study provides novel insights for the identification of a prognostic index with great clinical utility and therapeutic targets for KIRC treatments.

\section{Abbreviations}

KIRC, kidney renal clear cell carcinoma, APOA1, Apolipoprotein A1, RHWU, Renmin Hospital of Wuhan University, TCGA, The Cancer Genomic Atlas, GSEA, Gene Set Enrichment Analysis, HPA, The Human Protein Atlas, OS, overall survival, DFS, disease-free survival, $\mathrm{HR}$, hazard ratio, $95 \% \mathrm{Cl}, 95 \%$ confidence index; AUC, area under the curve.

\section{Declarations}

\section{Ethics approval and consent to participate}

The study outline was reviewed and smoothly approved by the ethics committee of the Renmin Hospital of Wuhan University.

\section{Consent for publication}

Not applicable.

\section{Availability of data and materials}

The dataset used during the study are available from the corresponding author on a reasonable request.

\section{Competing interests}

The authors declare that they have no competing interests.

\section{Funding}

Not applicable.

\section{Authors' contributions}

XLP performed data curation, formal analysis, funding acquisition, project administration, supervision, validation, visualizationv and writing - review \& editing. GGX and JTL performed data curation, formal analysis and writing - original draft. $\mathrm{YGH}, \mathrm{ABH}, \mathrm{XW}$ and $\mathrm{RFL}$ assisted in data curation, methodology, reviewed the manuscript. All authors reviewed and approved the final manuscript.

\section{Acknowledgements}

Not applicable. 


\section{References}

1. Siegel RL, Miller KD, Jemal A. Cancer statistics. 2020. CA Cancer J Clin (2020) 70(1):7-30. doi: 10.3322/caac.21590. PubMed PMID: 31912902.

2. Bhatt JR, Finelli A. Landmarks in the diagnosis and treatment of renal cell carcinoma. Nat Rev Urol (2014) 11(9):517 - 25. doi: 10.1038/nrurol.2014.194. PubMed PMID: 25112856.

3. Atkins MB, Tannir NM. Current and emerging therapies for first-line treatment of metastatic clear cell renal cell carcinoma. Cancer Treat Rev (2018) 70:127 - 37. doi: 10.1016/j.ctrv.2018.07.009. PubMed PMID: 30173085.

4. Inamura K. Renal Cell Tumors: Understanding Their Molecular Pathological Epidemiology and the 2016 WHO Classification. Int J Mol Sci (2017) 18(10). doi: 10.3390/ijms18102195. PubMed PMID: 29053609.

5. Chen D, Chen W, Xu Y, Zhu M, Xiao Y, Shen Y, et al. Upregulated immune checkpoint HHLA2 in clear cell renal cell carcinoma: a novel prognostic biomarker and potential therapeutic target. J Med Genet. 2019;56(1):43-9. doi:10.1136/jmedgenet-2018-105454. PubMed PMID: 29967134.

6. Li P, Liu J, Li J, Liu P. DNA methylation of CRB3 is a prognostic biomarker in clear cell renal cell carcinoma. Mol Biol Rep. 2019;46(4):4377-83. doi:10.1007/s11033-019-04892-7. PubMed PMID: 31147860.

7. Cao H, Zhang J, Wang W. DAB2IP Plays Important Clinical Significance and Correlates With Immune Infiltration in Renal Cell Carcinoma. Technol Cancer Res Treat. 2020;19:1079204330. doi: 10.1177/1533033820936682. PubMed PMID: 32583730.

8. Ducroux C, Desilles JP, Mawhin MA, Delbosc S, Ho-Tin-Noe B, Ollivier V, et al. Protective Effect of ApoA1 (Apolipoprotein A1)-Milano in a Rat Model of Large Vessel Occlusion Stroke. Stroke. 2020;51(6):1886-90. doi:10.1161/STROKEAHA.119.027898. PubMed PMID: 32404037.

9. Chen W, Wu Y, Lu Q, Wang S, Xing D. Endogenous ApoA-I expression in macrophages: A potential target for protection against atherosclerosis. Clin Chim Acta (2020) 505:55 - 9. doi:

10.1016/j.cca.2020.02.025. PubMed PMID: 32092318.

10. Chen BD, Chen XC, Yang YN, Gao XM, Ma X, Huang Y, et al. Apolipoprotein A1 is associated with SYNTAX score in patients with a non-ST segment elevation myocardial infarction. Lipids Health Dis. 2019;18(1):159. doi:10.1186/s12944-019-1101-9. PubMed PMID: 31391051.

11. Aguirre-Portoles C, Feliu J, Reglero G, Ramirez DMA. ABCA1 overexpression worsens colorectal cancer prognosis by facilitating tumour growth and caveolin-1-dependent invasiveness, and these effects can be ameliorated using the BET inhibitor apabetalone. Mol Oncol. 2018;12(10):1735-52. doi:10.1002/1878-0261.12367. PubMed PMID: 30098223.

12. Sirnio P, Vayrynen JP, Klintrup K, Makela J, Makinen MJ, Karttunen TJ, et al. Decreased serum apolipoprotein A1 levels are associated with poor survival and systemic inflammatory response in colorectal cancer. Sci Rep. 2017;7(1):5374. doi:10.1038/s41598-017-05415-9. PubMed PMID: 28710487. 
13. Marinho AT, Lu H, Pereira SA, Monteiro E, Gabra H, Recchi C. Anti-tumorigenic and PlatinumSensitizing Effects of Apolipoprotein A1 and Apolipoprotein A1 Mimetic Peptides in Ovarian Cancer. Front Pharmacol. 2018;9:1524. doi:10.3389/fphar.2018.01524. PubMed PMID: 30745873.

14. Liu JX, Yuan Q, Min YL, He Y, Xu QH, Li B, et al. Apolipoprotein A1 and B as risk factors for development of intraocular metastasis in patients with breast cancer. Cancer Manag Res. 2019;11:2881-8. doi:10.2147/CMAR.S191352. PubMed PMID: 31114339.

15. Mao M, Wang X, Sheng H, Liu Y, Zhang L, Dai S, et al. A novel score based on serum apolipoprotein A-1 and C-reactive protein is a prognostic biomarker in hepatocellular carcinoma patients. Bmc Cancer. 2018;18(1):1178. doi:10.1186/s12885-018-5028-8. PubMed PMID: 30486825.

16. Zhang F, Xie Y, Ma X, Gu L, Li H, Li X, et al. Preoperative apolipoprotein B/A1 ratio is an independent prognostic factor in metastatic renal cell carcinoma. Urol Oncol. 2019;37(3):184-9. doi: 10.1016/j.urolonc.2018.11.010. PubMed PMID: 30509867.

17. Guo S, He X, Chen Q, Yang G, Yao K, Dong P, et al. The Effect of Preoperative Apolipoprotein A-I on the Prognosis of Surgical Renal Cell Carcinoma: A Retrospective Large Sample Study. Med (Baltim). 2016;95(12):e3147. D.0000000000003147. PubMed PMID: 27015197.. ) ). doi: 10.1097/M.

18. Goldman MJ, Craft B, Hastie M, Repecka K, McDade F, Kamath A, et al. Visualizing and interpreting cancer genomics data via the Xena platform. Nat Biotechnol. 2020;38(6):675-8. doi:10.1038/s41587-020-0546-8. PubMed PMID: 32444850.

19. Chandrashekar DS, Bashel B, Balasubramanya S, Creighton CJ, Ponce-Rodriguez I, Chakravarthi B, et al. UALCAN: A Portal for Facilitating Tumor Subgroup Gene Expression and Survival Analyses. Neoplasia. 2017;19(8):649-58. doi:10.1016/j.neo.2017.05.002. PubMed PMID: 28732212.

20. Vasaikar SV, Straub P, Wang J, Zhang B. LinkedOmics: analyzing multi-omics data within and across 32 cancer types. Nucleic Acids Res. 2018;46(D1):D956-63. doi:10.1093/nar/gkx1090. PubMed PMID: 29136207.

21. Uhlen M, Zhang C, Lee S, Sjostedt E, Fagerberg L, Bidkhori G, et al. A pathology atlas of the human cancer transcriptome. Science (2017) 357(6352). doi:10.1126/science.aan2507. PubMed PMID: 28818916.

22. Li T, Fan J, Wang B, Traugh N, Chen Q, Liu JS, et al. TIMER: A Web Server for Comprehensive Analysis of Tumor-Infiltrating Immune Cells. Cancer Res. 2017;77(21):e108-10. doi:10.1158/0008-5472.CAN17-0307. PubMed PMID: 29092952.

23. Chandrasekaran D, Sundaram S. N K, R P. Programmed Death Ligand 1; An Immunotarget for Renal Cell Carcinoma. Asian Pac J Cancer Prev. 2019;20(10):2951-7. doi:10.31557/APJCP.2019.20.10.2951. PubMed PMID: 31653140.

24. Kucharczyk J, Matrana MR, Santoni M, Massari F, Scarpelli M, Cheng L, et al. Emerging Immunotargets in Metastatic Renal Cell Carcinoma. Curr Drug Targets. 2016;17(7):771-6. doi:10.2174/1389450117666151209115753. PubMed PMID: 26648075.

25. Dunnick NR. Renal cell carcinoma: staging and surveillance. Abdom Radiol (NY) (2016) 41(6):107985. doi: 10.1007/s00261-016-0692-0. PubMed PMID: 26971340. 
26. Martinez-Lopez D, Camafeita E, Cedo L, Roldan-Montero R, Jorge I, Garcia-Marques F, et al. APOA1 oxidation is associated to dysfunctional high-density lipoproteins in human abdominal aortic aneurysm. Ebiomedicine. 2019;43:43-53. doi: 10.1016/j.ebiom.2019.04.012. PubMed PMID: 30982767.

27. Clarke CH, Yip C, Badgwell D, Fung ET, Coombes KR, Zhang Z, et al. Proteomic biomarkers apolipoprotein $\mathrm{A} 1$, truncated transthyretin and connective tissue activating protein III enhance the sensitivity of CA125 for detecting early stage epithelial ovarian cancer. Gynecol Oncol. 2011;122(3):548-53. doi: 10.1016/j.ygyno.2011.06.002. PubMed PMID: 21708402.

28. Moore LE, Fung ET, McGuire M, Rabkin CC, Molinaro A, Wang Z, et al. Evaluation of apolipoprotein A1 and posttranslationally modified forms of transthyretin as biomarkers for ovarian cancer detection in an independent study population. Cancer Epidemiol Biomarkers Prev. 2006;15(9):1641-6. doi:10.1158/1055-9965.EPI-05-0980. PubMed PMID: 16985025.

29. Liu Z, Xiao Y, Tang L, Jiang L, Wang Y, Zhang R, et al. Apolipoprotein A1 -75 G/A and + $83 \mathrm{C} / \mathrm{T}$ polymorphisms and renal cancer risk. Lipids Health Dis. 2015;14:143. doi:10.1186/s12944-0150132-0. PubMed PMID: 26537097.

30. Luo XL, Zhong GZ, Hu LY, Chen J, Liang Y, Chen QY, et al. Serum apolipoprotein A-I is a novel prognostic indicator for non-metastatic nasopharyngeal carcinoma. Oncotarget. 2015;6(41):4403748. doi:10.18632/oncotarget.5823. PubMed PMID: 26503474.

31. Wang XP, Li XH, Zhang L, Lin JH, Huang H, Kang T, et al High level of serum apolipoprotein A-I is a favorable prognostic factor for overall survival in esophageal squamous cell carcinoma. Bmc Cancer (2016) 16:516. doi: 10.1186/s12885-016-2502-z. PubMed PMID: 27444612.

32. Massari F, Santoni M, Ciccarese C, Santini D, Alfieri S, Martignoni G, et al. PD-1 blockade therapy in renal cell carcinoma: current studies and future promises. Cancer Treat Rev. 2015;41(2):114-21. doi: 10.1016/j.ctrv.2014.12.013. PubMed PMID: 25586601.

33. Iqbal AJ, Barrett TJ, Taylor L, McNeill E, Manmadhan A, Recio C, et al. Acute exposure to apolipoprotein $\mathrm{A} 1$ inhibits macrophage chemotaxis in vitro and monocyte recruitment in vivo. Elife (2016) 5. doi:

10.7554

/eLife.15190. PubMed PMID: 27572261.

34. Mao J, Liu W, Wang Y. Apolipoprotein A-l expression suppresses COX-2 expression by reducing reactive oxygen species in hepatocytes. Biochem Biophys Res Commun. 2014;454(3):359-63. doi:10.1016/j.bbrc.2014.10.094. PubMed PMID: 25451254.

35. Kelsey R. Kidney cancer: PDL1 as a biomarker in high-risk RCC. Nat Rev Urol. 2018;15(4):202. doi:10.1038/nrurol.2018.19. PubMed PMID: 29434371.

36. Erlmeier F, Weichert W, Schrader AJ, Autenrieth M, Hartmann A, Steffens S, et al Prognostic impact of PD-1 and its ligands in renal cell carcinoma. Med Oncol(2017) 34(6):99. doi: 10.1007/s12032-0170961-y. PubMed PMID: 28432616. 
37. Joseph RW, Parasramka M, Eckel-Passow JE, Serie D, Wu K, Jiang L, et al. Inverse association between programmed death ligand 1 and genes in the VEGF pathway in primary clear cell renal cell carcinoma. Cancer Immunol Res. 2013;1(6):378-85. doi:10.1158/2326-6066.CIR-13-0042. PubMed PMID: 24778130.

38. Casillas-Munoz F, Valle Y, Munoz-Valle JF, Martinez-Fernandez DE, Reynoso-Villalpando GL, FloresSalinas HE, et al. APOA1 and APOB polymorphisms and apolipoprotein concentrations as biomarkers of risk in acute coronary syndrome: Relationship with lipid-lowering therapy effectiveness. Med Clin (Barc). 2018;151(1):1-7. doi:10.1016/j.medcli.2017.07.026. PubMed PMID: 28992985.

39. Chinello C, Stella M, Piga I, Smith AJ, Bovo G, Varallo M, et al. Proteomics of liquid biopsies: Depicting RCC infiltration into the renal vein by MS analysis of urine and plasma. J Proteomics. 2019;191:29-37. doi: 10.1016/j.jprot.2018.04.029. PubMed PMID: 29689304.

\section{Figures}



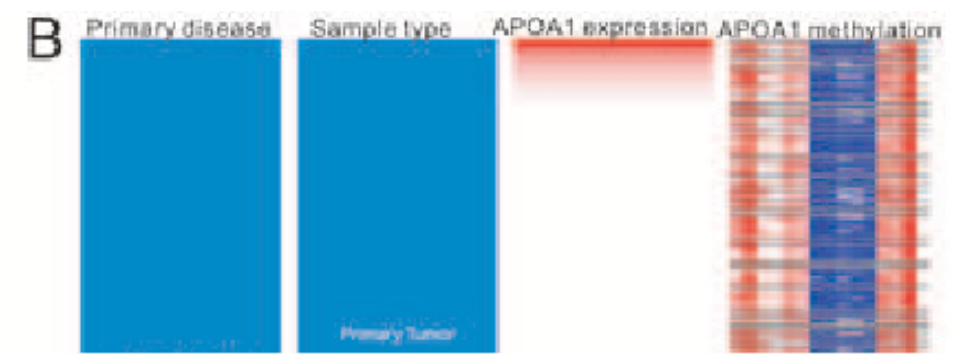

\section{Figure 1}

Expression and methylation of APOA1 in KIRC tissues and the normal renal tissues. A. UALCAN website was mined to compare the difference of APOA1 mRNA expression in normal tissues $(\mathrm{N}=72)$ and KIRC tissues ( $N=533)$, and the student $t$ test revealed no statistical significance. B. UCSC Xena webpage was browsed to generate the heatmap of APOA1 mRNA expression and DNA methylation in KIRC dataset. C. Distribution of APOA1 DNA CpG site among primary tumors, normal tissues and additional-New primary tumors was obtained from UCSC Xena webpage. 

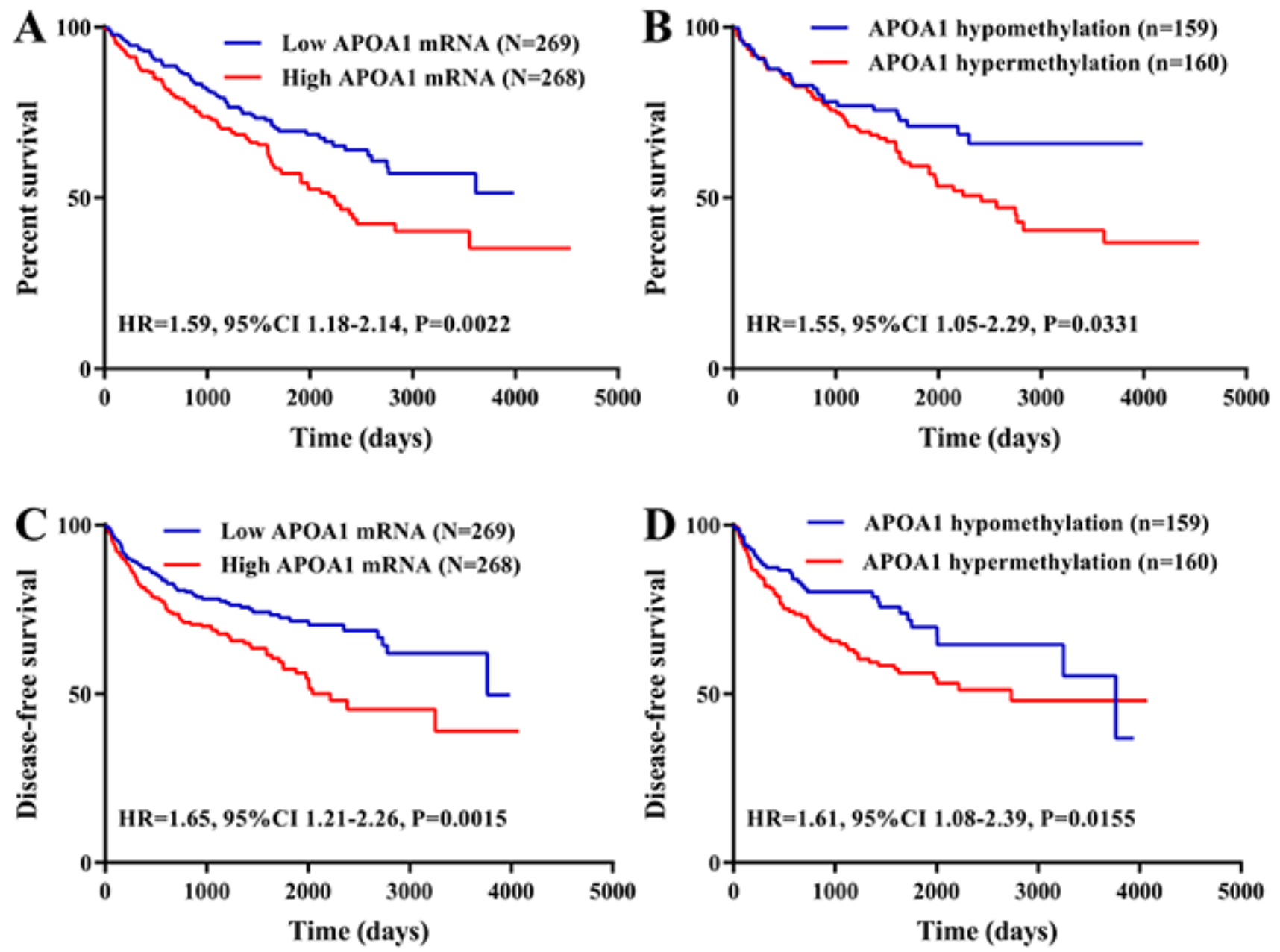

Figure 2

Survival analyses comparing low and high APOA1 mRNA/methylation in KIRC patients. KIRC patients with low levels of APOA1 mRNA ( $N=269)$ exhibited longer overall survival $(A)$ and disease-free survival $(B)$ time than those with high levels of APOA1 mRNA $(\mathrm{N}=268)$. KIRC patients with APOA1 hypermethylation $(\mathrm{N}=159)$ exhibited longer overall survival (C) and disease-free survival (D) time than those with APOA1 hypomethylation $(\mathrm{N}=160)$.

\section{Figure 3}

Representative images stained with APOA1 antibody (CAB016778; HPA046715). A-D The normal tissues. E-F KIRC tissues. HPA046715 is a polyclonal antibody derived from rabbits and produced by SigmaAldrich, and CAB016778 is a monoclonal antibody derived from mice and produced by BioPorto Diagnostics A/S. 


\section{Figure 4}

Diagnostic and prognostic significance of serum APOA1 protein in KIRC patients. A. Concentrations of serum APOA1 protein in healthy individuals are much higher than that in KIRC patients $(P<0.001)$. B. ROC curve of serum APOA1 protein for the identification of KIRC patients from normal controls. C. KaplanMeier plot of serum APOA1 protein among 91 cases of KIRC patients. D. Forest plot of the multivariable analysis of overall survival in KIRC patients.

\section{Figure 5}

Relationships between levels of APOA1 protein and common clinical features in 91cases of KIRC patients. A age ( $P=0.2867)$; . gender $(P=0.1305)$; . tumor site $(P=0.1633)$; $\mathbf{D}$. histological grade $(P=0.0047) ; E$. T stage $(P<0.0001) ;$ F. N stage $(P=0.4939) ; G$. M stage $(P=0.0001) ; H$. TNM stage $(P=0.0138)$.

\section{Figure 6}

Co-expressed genes with APOA1 and Go analysis of these genes in KIRC based on LinkedOmics database. A. Volcano plot of the genes relevant to APOA1 expression in KIRC tissues. B. Significant genes positively correlated with APOA1 expression. C. Significant genes negatively correlated with APOA1 expression.

\section{Supplementary Files}

This is a list of supplementary files associated with this preprint. Click to download.

- Supportinginformation.doc

- renamedd671e.xls 